\title{
Resonance, Metastability and Blow up in FPU
}

\author{
D. Bambusi, A. Ponno
}

14.12 .05

\begin{abstract}
We consider the FPU model with nonlinearity starting with terms of order $n \geq 3$. We compute the resonant normal form in the region where only one low frequency modes is excited and deduce rigorous results on the correspondence between the dynamics of the normal form and that of the complete system. As $n$ varies, we give a criterion in order to deduce whether the FPU phenomenon (formation of a metastable packet of modes) is present or not. The criterion is that, if the normal form equation has smooth solutions then the FPU phenomenon is present, while it is absent if the solutions of the normal form equations have blow up in a finite time. In particular the phenomenon should be present for $n \leq 6$ and absent for $n \geq 7$.
\end{abstract}

\section{Introduction}

In the present contribution some analytic results on the Fermi-Pasta-Ulam (FPU) problem are presented. Our purpose is to use the methods of rigorous perturbation theory for infinite dimensional Hamiltonian systems to explain some of the features of the FPU phenomenology. In particular we have the following results

(1) For low energy and long wavelength initial data, canonical perturbation theory allows to put the Hamiltonian of the system in resonant normal form up to a small remainder. The equations of motion of the normal form consist of two partial differential equations (PDE's) which describe well the dynamics of the system within a certain time-scale.

(2) In the case of the so-called FPU $\alpha$-model, the normal form equations are two uncoupled Kortweg-de Vries (KdV) equations. Thus all the remarkable features of the $\mathrm{KdV}$ equation turn out to pertain, in an approximate way, also to the FPU model. In particular the $\alpha$-model will behave as an integrable system over the time-scale of validity of the normal form. We emphasize that, as predicted in [Pon03], such a time-scale is of order $\epsilon^{-3 / 4}, \epsilon$ being the specific energy (i.e. the energy per degree of freedom) of the system. Such a time-scale has to be considered as short in connection with the problem of thermalization. Indeed, thermalization, at least in 
dimension one, seems to take place on a much longer time-scale, namely a Nekhoroshev-like stretched exponential of $1 / \epsilon$. So the first conclusion, at least from a rigorous point of view, is that $\mathrm{KdV}$ is relevant for the dynamics of the FPU (as heuristically predicted since the pioneering works of Zabusky and Kruskal [ZK65]) on short, power law time-scales, as opposed to the longer, exponential ones over which one can observe relaxation to equilibrium.

(3) The KdV equation allows to explain (rigorously), in the case of the $\alpha-$ chain, the phenomenon of formation of the packet [FPU65, GS72, KBL03, BGG04, BGP04, GPP05], namely the fact (first observed by Fermi Pasta and Ulam themselves) that, if one low frequency mode is initially excited, then the energy quickly flows to a small packet of modes whose energy, in time average, decreases exponentially with the mode index. The packet turns out to be stable over the time scale covered by the normal form, namely $\epsilon^{-3 / 4}$.

(4) The situation of having a resonant normal form which is integrable seems to be quite exceptional: indeed, if one introduces action angle variables one sees that the normalization procedure consists in eliminating just one among infinitely many angles; thus the system in normal form is in principle expected to have only one integral of motion independent of the energy. Instead, the KdV has a complete set (infinitely many) of integrals of motion in involution! We are thus naturally led to study the $\beta$-model and systems with perturbations of higher order, in order to check the occurrence and the relevance of integrability. The normal form of the $\beta$-model turns out to consist of two uncoupled modified $\mathrm{KdV}(\mathrm{mKdV})$ equations which are integrable too. The same qualitative behavior of the $\alpha$-model is thus expected also in the $\beta$-model (a rigorous proof is still missing due to the lack of a deeper knowledge of the $\mathrm{mKdV}$ equation).

(5) The situation changes with higher order FPUs where the normal form equations we get are higher order generalized KdV's (gKdV) that are no longer integrable. One is thus naturally led to ask whether the FPU phenomenon persists in such cases, and whether the integrability of the normal form plays a fundamental role or not. We think that the answer is no, and that there should be a weaker property entraining the formation of a metastable packet of modes. We guess that such a property actually is related to the smoothness of the solutions of the Cauchy problem of the normal form PDEs. In particular if the solutions of the PDEs under investigation have blow up in a finite time we guess that metastability should be lost, while we think that the phenomenon of formation of a metastable packet of modes should be present in all models that do not display blow up. We also give a heuristic argument for that.

For the case of one-dimensional FPU chains it turns out that the metastable packet is expected to exist in the case of nonlinearity of degree less than or equal to 5, while it is expected not to exist when the nonlinearity has 
degree higher than or equal to 7 . The case of nonlinearity of order 6 is critical and therefore the existence of the packet strongly depends, in principle, on the initial conditions.

\section{Normal Form}

Consider the Hamiltonian system

$$
\begin{aligned}
& H(q, p)=\sum_{j=-N}^{N-1} \frac{p_{j}^{2}}{2}+U\left(q_{j+1}-q_{j}\right), \\
& U(x)=\frac{x^{2}}{2}+\frac{x^{n}}{n}, \quad n \geq 3 \\
& q_{j+2 N}=q_{j}, \quad p_{j+2 N}=p_{j},
\end{aligned}
$$

describing a periodic chain of $2 N$ particles interacting through nonlinear springs. The canonical variables are $q=\left(q_{-N}, \ldots, q_{N-1}\right), p=\left(p_{-N}, \ldots, p_{N-1}\right)$. Hamiltonians of the form (2.1) were first introduced and studied by Fermi, Pasta and Ulam (FPU) in the case $n=3,4$; such model Hamiltonians are commonly referred to as FPU models (in the case $n=3$ and $n=4$ one refers to the $\alpha$-model and $\beta$-model, respectively). Due to the periodic boundary conditions (2.3), the total linear momentum of the system is preserved. So one can restrict oneself to the case $\sum_{j} p_{j}=\sum_{j} q_{j}=0$.

The equations of motion associated to (2.1) are given by

$$
\ddot{q}_{j}=q_{j+1}+q_{j-1}-2 q_{j}+\left(q_{j+1}-q_{j}\right)^{n-1}-\left(q_{j}-q_{j-1}\right)^{n-1}
$$

We are interested in initial data in which only one Fourier mode is excited, say the one with wave number $k_{0} \ll N$.

Remark 2.1. In this case the dynamics is equivalent to the dynamics of a shorter FPU chain; in particular, if $k_{0}$ divides $N$ it turns out that the dynamics is equivalent to that of a chain of length $2 N / k_{0}$, in which only the first Fourier mode is excited. More precisely, the solution lies on an invariant submanifold on which the Fourier modes with index which is not an integer multiple of $k_{0}$ (modulo $N$ ) are exactly zero (see [Rin03]).

We will use as a small parameter

$$
\mu:=\frac{k_{0}}{N} .
$$

To begin with, we construct a canonical transformation setting the system in normal form in the region of long wavelength states. We rewrite the FPU system in terms of the new variables $r_{j}$ defined by

$$
r_{j}:=q_{j}-q_{j-1}, \sum_{j} r_{j}=0 ;
$$


the change of variables $q \rightarrow r$ is well defined and invertible. Introducing also the discrete Laplacian $\Delta_{1}$ defined by

$$
\left(\Delta_{1} r\right)_{j}:=r_{j+1}+r_{j-1}-2 r_{j},
$$

the FPU equations take the form

$$
\ddot{r}=\Delta_{1}\left(r+r^{n-1}\right) .
$$

We introduce now an interpolating function $r=r(x, t)$ for the sequence $r_{j}$, namely a (smooth) function with the property that the sequence

$$
r_{j}(t) \equiv r(j, t)
$$

fulfills the FPU equations (2.8). Moreover we will assume that the function $r(x, t)$ is $2 N / k_{0} \equiv 2 / \mu$ periodic and has zero average. Thus we postulate that the function $r$ fulfills equation (2.8) with an obvious extension of the definition of $\Delta_{1}$ to smooth functions.

We remark that up to now we did essentially nothing: the continuous system we got is equivalent to the original FPU system.

Since we are interested in states with long wavelength and small amplitude we will look for solutions of the form

$$
r(x, t)=\mu^{\frac{2}{n-2}} u(\mu x, \mu t)
$$

it turns out that such a re-scaling gives a correct balance between dispersive and nonlinear terms in the equations of motion (see below). It has to be remarked at this stage that by the choice $(2.10)$ one links the small parameter $\mu$ defined by $(2.5)$ to the specific energy of the system (energy per degree of freedom). Indeed, from

$$
\epsilon \equiv \frac{E}{2 N} \sim \frac{1}{2 N} \sum_{n} r_{n}^{2}=\mu^{\frac{4}{n-2}} \frac{1}{2 N} \sum_{n} u^{2}(\mu n)
$$

it follows that

$$
\mu \sim \epsilon^{(n-2) / 4}
$$

We remark that if $u$ is a smooth function (e.g. analytic) then it turns out that the Fourier coefficients of $r$ and therefore of the original variables of the FPU model will decay fast (e.g. exponentially) with the index $k$. Correspondingly, one expects that it should be possible to approximate the frequencies of the FPU with their low mode expansion. Indeed, when $k / N$ is small, one has

$$
\omega(k) \equiv 2 \sin \left(\frac{k \pi}{2 N}\right)=\frac{k \pi}{N}-\frac{k^{3} \pi^{3}}{24 N^{3}}+O\left((k / N)^{5}\right) .
$$

By substituting (2.10) into (2.8) one gets the equation of motion for $u$, namely

$$
u_{\tau \tau}=\mu^{-2} \Delta_{\mu}\left(u+\mu^{2} u^{n-1}\right) .
$$


Here $\Delta_{\mu}$ is the difference operator

$$
\left(\Delta_{\mu} u\right)(y):=u(y+\mu)+u(y-\mu)-2 v(y) .
$$

Notice that equation (2.13) is written in terms of the rescaled variables $y=\mu x$ and $\tau=\mu t$ introduced in (2.10); moreover, since the function $r(x)$ is $2 / \mu$ periodic, then the function $u(y)$ is 2-periodic. Equation (2.13) is Hamiltonian with Hamiltonian function

$$
K(u, v)=\int_{-1}^{1}\left(\frac{-v \Delta_{\mu} v}{2 \mu^{2}}+\frac{u^{2}}{2}+\frac{\mu^{2} u^{n}}{n}\right) d y,
$$

where $v$ is the variable canonically conjugated to $u$. From now on we will study the system (2.15).

Inserting the formal expansion of the operator $\Delta_{\mu}(2.14)$, given by

$$
\frac{\Delta_{\mu}}{\mu^{2}}=\partial_{y}^{2}+\frac{\mu^{2} \partial_{y}^{4}}{12}+O\left(\mu^{4}\right),
$$

into the Hamiltonian (2.15), one gets

$$
K=H_{0}+\mu^{2} P+\mathcal{R}_{1},
$$

where

$$
\begin{array}{r}
H_{0}(u, v):=\int_{-1}^{1}\left[\frac{v\left(-\partial_{y}^{2} v\right)+u^{2}}{2}\right] d y=\sum_{k \neq 0} \frac{\hat{u}_{k}^{2}+(k \pi)^{2} \hat{v}_{k}^{2}}{2}, \\
P(u, v):=\int_{-1}^{1}\left[-\frac{v \partial_{y}^{4} v}{24}+\frac{u^{n}}{n}\right] d y,
\end{array}
$$

and $\mathcal{R}_{1} \sim O\left(\mu^{4}\right)$ is the remainder of the expansion. In (2.18) $\hat{u}_{k}$ and $\hat{v}_{k}$ are the Fourier coefficients of $u$ and $v$, respectively. More precisely, the Fourier coefficients of a (zero average) function $u(y)$ are defined here as

$$
u(y)=\frac{1}{\sqrt{2}} \sum_{k>0}\left[\hat{u}_{k} \cos (\pi k y)+\hat{u}_{-k} \sin (\pi k y)\right]
$$

and clearly $\hat{v}_{k}$ is the momentum conjugated to $\hat{u}_{k}$.

Remark 2.2. The equations of motion of $H_{0}+\mu^{2} P$, in second order form, are

$$
u_{\tau \tau}=\left[u+\mu^{2} \frac{1}{12} u_{y y}+\mu^{2} u^{n-1}\right]_{y y},
$$

which is a generalized Boussinesq ( $\mathrm{gB}$ ) equation, sometimes considered as a starting point in approaching the FPU problem. In particular the equation (2.21) has been shown to be integrable in the case $n=3$ (even if the actual integration is non trivial); in the other cases the equation is non integrable. 
The strategy for studying the dynamics of (2.17) is well known from the theory of finite dimensional systems: substitute $P$ with its average $\langle P\rangle$ with respect to the flow of the Hamiltonian vector field $X_{H_{0}}$, and study the dynamics of this system, then use some rigorous method in order to prove that the solutions of the averaged system, namely of the system

$$
H_{0}+\mu^{2}\langle P\rangle
$$

are close to the solutions of the original one at least over some long time-scale.

We start now giving a precise definition of $\langle P\rangle$. So we consider the equations of motion of $H_{0}$, i.e.,

$$
\left\{\begin{array} { c } 
{ u _ { \tau } = - \partial _ { y } ^ { 2 } v } \\
{ v _ { \tau } = - u }
\end{array} \Longleftrightarrow \left\{\begin{array}{c}
\frac{d \hat{u}_{k}}{d \tau}=\hat{v}_{k} \\
\frac{d \hat{v}_{k}}{d \tau}=-(\pi k)^{2} \hat{u}_{k}
\end{array} .\right.\right.
$$

Denote by $(u, v)=\Psi^{\tau}\left(u_{0}, v_{0}\right)$ the solution of the Cauchy problem with initial datum $\left(u_{0}, v_{0}\right)$, which in terms of Fourier coefficients is given by

$$
\begin{gathered}
\hat{u}_{k}(\tau)={\widehat{\left(u_{0}\right)}}_{k} \cos (\pi k \tau)+\frac{{\widehat{\left(v_{0}\right)}}_{k}}{k \pi} \sin (\pi k \tau), \\
\hat{v}_{k}(\tau)={\widehat{\left(v_{0}\right)}}_{k} \cos (\pi k \tau)-k \pi \widehat{\left(u_{0}\right)} \sin (\pi k \tau),
\end{gathered}
$$

and in the $x$ space is still more complicated. In the following we will show that there is a third representation in which the computations are particularly simple. In order to keep in mind that all we will do is completely independent of the coordinate system, we will use the abstract notation $z$ for a phase space point, namely for the pair $(u, v)$. Correspondingly, the flow of $H_{0}$ will be denoted by $\Psi^{\tau}(z)$

Definition 2.3. The average of $P$ is defined by

$$
\langle P\rangle(z):=\frac{1}{2} \int_{0}^{2} P\left(\Psi^{\tau}(z)\right) d \tau
$$

Remark 2.4. In the jargon of Birkhoff normal forms one usually says that $\langle P\rangle$ is the resonant part of $P$, and indeed, when expressed in the complex variables usually employed in order to solve the homological equation it contains all (and only) the resonant monomials present in $P$.

As anticipated above the explicit computation of $\langle P\rangle$ (which is very complicated in Fourier space) is simpler in different variables that we presently introduce. Consider the non canonical change of variables

$$
\xi:=\frac{u+v_{y}}{\sqrt{2}}, \quad \eta:=\frac{u-v_{y}}{\sqrt{2}} .
$$

Correspondingly the equations of motion take the form

$$
\frac{d z}{d \tau}=J \nabla H(z), \Longleftrightarrow\left(\xi_{\tau}=-\partial_{y} \frac{\delta H}{\delta \xi}, \eta_{\tau}=\partial_{y} \frac{\delta H}{\delta \eta}\right),
$$


where $\nabla H$ denotes the $L^{2}$ gradient of $H(\xi, \eta)$ and the Poisson tensor is now defined by

$$
J=\left(\begin{array}{cc}
-1 & 0 \\
0 & 1
\end{array}\right) \partial_{y} .
$$

In the variables $(\xi, \eta)$ the various parts of the Hamiltonian take the form

$$
\begin{aligned}
H_{0}(\xi, \eta) & =\int_{-1}^{1} \frac{\xi^{2}+\eta^{2}}{2} d y \\
P(\xi, \eta) & =\int_{-1}^{1}\left[-\frac{\left[\partial_{y}(\xi-\eta)\right]^{2}}{48}+\frac{(\xi+\eta)^{n}}{n 2^{n / 2}}\right] d y,
\end{aligned}
$$

and in particular the equations of motion of $H_{0}$ and the flow $\Psi^{\tau}$ assume the simple form

$$
\left[\xi_{\tau}=-\xi_{y}, \quad \eta_{\tau}=\eta_{y}\right] \Longleftrightarrow\left[\xi(y, \tau)=\xi_{0}(y-\tau), \eta(y, \tau)=\eta_{0}(y+\tau)\right] .
$$

It is now easy to obtain the following

Proposition 2.5. In the variables $\xi, \eta$ the average of the perturbation is given by

$$
\langle P\rangle(\xi, \eta)=-\int_{-1}^{1}\left[\frac{\left(\xi_{y}\right)^{2}+\left(\eta_{y}\right)^{2}}{48}\right]+\frac{1}{2^{n / 2} n} \sum_{l=0}^{n} C_{l}^{n}\left[\xi^{l}\right]\left[\eta^{n-l}\right]
$$

In the above expression, $C_{l}^{n} \equiv n ! /(l !(n-l) !)$ while $\left[f^{j}\right] \equiv \int_{0}^{L} f^{j} d x / 2$; we will refer to the latter as to the moment of $f$ of order $j$, or simply the $j$-th moment of $f$.

For the proof see [BP05, PB05].

The equations of motion associated to $H_{0}+\mu^{2}\langle P\rangle$ are

$$
\left\{\begin{array}{l}
\xi_{\tau}=-\xi_{y}-\mu^{2}(1 / 24) \xi_{y y y}-\mu^{2}\left(1 / 2^{n / 2}\right) \sum_{l=1}^{n-1} C_{l}^{n-1}\left[\eta^{n-l-1}\right]\left(\xi^{l}\right)_{y} \\
\eta_{\tau}=\eta_{y}+\mu^{2}(1 / 24) \eta_{y y y}+\mu^{2}\left(1 / 2^{n / 2}\right) \sum_{l=1}^{n-1} C_{l}^{n-1}\left[\xi^{n-l-1}\right]\left(\eta^{l}\right)_{x}
\end{array}\right.
$$

These are generalized Kortweg-de Vries (gKdV) equations.

One can easily check that for $n=3$ and $n=4$ they yield, respectively, the $\mathrm{KdV}$ and the modified KdV equation (see below), which are both integrable. Moreover it is now clear that they appear here as the resonant normal forms of the corresponding FPU models ${ }^{1}$.

Notice that, as a consequence of averaging, the two second order momenta of $\xi$ and $\eta$ are constants of motion for system (2.34). Moments of order greater than two will be time-dependent, and as a consequence the above equations are actually coupled and of integro-differential type for $n \geq 6$ (as we will show below the case $n=5$ is a special one).

\footnotetext{
${ }^{1}$ For an interesting related result see [Rin01]
} 


\section{Metastability or Blow up}

In this section we discuss the dynamics of the normal-form equations (2.34) from a qualitative point of view.

\section{$3.1 n=3: \alpha-$ model}

To begin with, let us consider the case $n=3$, and report the results obtained in [BP05]. The equations of motion are

$$
\begin{aligned}
\xi_{\tau} & =-\xi_{y}-\mu^{2} \frac{1}{24} \xi_{y y y}-\mu^{2} \frac{1}{2 \sqrt{2}}\left(\xi^{2}\right)_{y}, \\
\eta_{\tau} & =\eta_{y}+\mu^{2} \frac{1}{24} \eta_{y y y}+\mu^{2} \frac{1}{2 \sqrt{2}}\left(\eta^{2}\right)_{y},
\end{aligned}
$$

i.e. two uncoupled $\mathrm{KdV}$ equations in translating frames (to the right for the first equation and to the left for the second one). Their dynamics is very well understood, and in particular it is known that any solution of the Cauchy problem is almost periodic and the solution has the same regularity as the initial datum. One can easily conclude from the theory of [KP03] that if one initially gives energy only to the first Fourier mode then, up to infinite times, the solution remains analytic, and therefore the energy remains exponentially localized in the first Fourier modes. Thus, corresponding to initial data on a low frequency Fourier mode one will see that, on a time scale of the order of $\mu^{-3}$, the energy will flow to a small packet of modes which oscillate with an amplitude exponentially decreasing with the mode index, and this situation will last forever. Thus, in KdV there is a formation of a small packet $\grave{a}$ la FPU which is stable for infinite times (the time averages of the mode-energies relax).

In order to get conclusions on the dynamics of the FPU one needs a precise relation between the KdV and the FPU. From the fact that the KdV is a first order normal form of the system one expects that the solutions of KdV describe well the FPU only up to times of order $\mu^{-3} \sim \epsilon^{-3 / 4}$. This was proved in [BP05] by a rigorous theory that will be summarized in section 4 (see theorem 4.4 below for a precise statement).

Afterwords one can expect that higher order corrections come into play and modify the dynamics. However, one has to remark that the $\mathrm{KdV}$ is an integrable system which is nondegenerate (in KAM sense) and therefore one expects higher order corrections not to modify qualitatively the dynamics. In the spirit of Nekhoroshev's theory, the dynamics of the KdV should be unchanged up to times exponentially growing with a power of $\epsilon^{-1}$. The small packet of modes which was quickly formed should persist for exponentially long times and represent, as a consequence, a metastable state of the system. This was actually verified numerically in [BGP04].

It is worth mentioning that this phenomenon is related to two properties, namely the fact that the linearized FPU is resonant (more precisely the dispersion relation is approximatively linear for low frequencies), and the very 
surprising fact that the first order normal form is integrable. Indeed, if the linearized system were nonresonant, the perturbation would not be able to efficiently spread energy among the modes, thus preventing the formation of the packet of modes.

\section{$3.2 \mathrm{n}=4: \beta-$ model}

In the case $n=4$ the situation is similar to the case $n=3$. The equations of motion have the form

$$
\begin{aligned}
& \xi_{\tau}=-\left(1+\frac{3 \mu^{2}}{4}\left[\eta^{2}\right]\right) \xi_{y}-\frac{\mu^{2}}{24} \xi_{y y y}-\frac{\mu^{2}}{4}\left(\xi^{3}\right)_{y} \\
& \eta_{\tau}=\left(1+\frac{3 \mu^{2}}{4}\left[\xi^{2}\right]\right) \eta_{y}+\frac{\mu^{2}}{24} \eta_{y y y}+\frac{\mu^{2}}{4}\left(\eta^{3}\right)_{y}
\end{aligned}
$$

which, taking into account the conservation of the momenta of order two, reduce to two uncoupled $\mathrm{mKdV}$ equations in translating frames.

Here however, since the $\mathrm{mKdV}$ is less studied we are unable to provide a precise theorem even for the formation of a packet which is stable over a time scale of order $\mu^{-3}$. However we think that this is only a technical problem, and indeed numerical computations display a behavior very similar to that of the $\alpha$-FPU [BGP04].

\section{3 $n=5: \gamma$-model}

It is interesting to consider explicitly also the case $n=5$. In such a case the equations of motion (2.34) read

$$
\begin{aligned}
& \xi_{\tau}=-\left(1+\frac{\mu^{2}\left[\eta^{3}\right]}{\sqrt{2}}\right) \xi_{y}-\frac{\mu^{2}}{24} \xi_{y y y}-\frac{3 \mu^{2}}{2 \sqrt{2}}\left[\eta^{2}\right]\left(\xi^{2}\right)_{y}-\frac{\mu^{2}}{4 \sqrt{2}}\left(\xi^{4}\right)_{y} \\
& \eta_{\tau}=\left(1+\frac{\mu^{2}\left[\xi^{3}\right]}{\sqrt{2}}\right) \eta_{y}+\frac{\mu^{2}}{24} \eta_{y y y}+\frac{3 \mu^{2}}{2 \sqrt{2}}\left[\xi^{2}\right]\left(\eta^{2}\right)_{y}+\frac{\mu^{2}}{4 \sqrt{2}}\left(\eta^{4}\right)_{y}
\end{aligned}
$$

Notice that in this case the (time dependent) momenta of third order appear on the r.h.s. of the two equations. By the change of variables

$$
\xi(y, \tau)=\xi^{\prime}(y-a(\tau), \tau), \eta(y, \tau)=\eta^{\prime}(y+b(\tau), \tau)
$$

where

$$
\frac{d a}{d \tau} \equiv 1+\frac{\mu^{2}\left[\eta^{3}\right](\tau)}{\sqrt{2}} \quad, \quad \frac{d b}{d \tau} \equiv 1+\frac{\mu^{2}\left[\xi^{3}\right](\tau)}{\sqrt{2}},
$$

the equations (3.5)-(3.6) become two uncoupled KdV equations of fourth order.

\section{4 $n \geq 6$ : higher models}

In the case $n \geq 6$ the normalized equations consist of a pair of coupled integrodifferential gKdV equations. Their behavior can be studied heuristically using for 
example scaling arguments and one can show that, if one starts with analytic (in space) initial data having a pole at a certain distance from the real axis, then the corresponding solution is expected to remain analytic over $\mathbb{R}$ if $n \leq 5$, while the pole is expected to reach the real axis if $n \geq 7$, the case $n=6$ being "critical". Since the whole behavior seems to depend only on the homogeneity degree of the nonlinearity and on the fact that the momenta of order two are conserved, it is useful to use a model problem where a lot is known, namely the standard gKdV

$$
\xi_{t}=\xi_{y y y}+\left(\xi^{n-1}\right)_{y} .
$$

Here (at least on unbounded domains) it is known [MM02] that corresponding to smooth initial data there are two possibilities: (1) the solution remains smooth for all times or (2) the $H^{1}$ (energy) norm of the solution becomes infinite at a finite time. The situation depends on the value of $n$. If $n \leq 5$ the solution is smooth for all times, if $n \geq 7$ every solution is expected to blows up in finite time, and finally, if $n=6$ then the behavior of the solution depends on the initial datum and there are rigorous results on the existence of blow up solutions [Mer01].

As a consequence, one might conjecture the existence of a corresponding situation for the FPU: if $n \leq 5$ a metastable packet of modes is expected to exists, if $n \geq 7$ the FPU phenomenon is expected to disappear, and finally, the case $n=6$ is critical in the sense that the packet will exist only for some initial data.

It is important to remark that in the case $n=5$ the normal form equations (3.5)-(3.6) are not integrable. However, according to our conjecture one would expect to observe a FPU phenomenology similar to the cases $n=3$, 4 , where the normal form equations are integrable. This fact forces to look for a mechanism weaker than integrability to explain the FPU paradox. The stability of the soliton solutions of (3.7) for $n \leq 5$ and their blowing-up for $n \geq 7$ suggest that a sort of Soliton Turbulence, analogous to the one proposed by Zakharov in the context of the NLS equations, might be the relevant phenomenon in FPU.

From a physical point of view, stable, space-localized solitary wave structures, trap the energy on a spatial scale which is lower bounded. As a consequence, in Fourier space the energy cannot flow above some critical wavenumber. On the contrary, in the supercritical cases $n \geq 7$, to the collapse in real space of the solitary wave structures there corresponds energy injection at any Fourier wavenumber, which we interpret as the mechanism triggering off the approach to equipartition in a short time.

In conclusion, we conjecture the existence of a critical degree of nonlinearity, $n_{c}=6$, in system (2.4), below which the FPU phenomenology (metastability of partial equipartition states) should be possible, and above which it could disappear. The relevant quantities characterizing the metastable state in the subcritical cases $(n \leq 5)$, are the time-scale $T_{1}$ of formation of the metastable state (consisting of a packet of modes sharing the energy) and almost equipartited, and the size $\Delta k / N$ of such a packet in Fourier space. From the scaling previously introduced in terms of the small parameter $\mu$ and from its link with 
the specific energy given in (2.11), one easily gets

$$
T_{1} \sim \mu^{-3} \sim \epsilon^{-\frac{3(n-2)}{4}},
$$

and

$$
\Delta k / N \sim \mu \sim \epsilon^{\frac{n-2}{4}},
$$

which generalize to the case of arbitrary $n$ those introduced in [Pon03].

\section{Rigorous results}

We give here the precise relation between the FPU ant its normal form (2.34) and deduce some rigorous results on the formation of the packet.

First of all we have to introduce a norm in order to measure the error between the correct equations and the equations in normal form. This is conveniently done in terms of Fourier coefficients. Moreover, since we are interested in exponentially localized packets we will use a norm that controls the Fourier coefficients with an accuracy growing exponentially with the index.

Definition 4.1. Having fixed two positive constants $s, \sigma$ consider the Hilbert space $\ell_{\sigma, s}^{2}$ of the complex sequences $v \equiv\left\{v_{k}\right\}_{k \in \mathbb{Z}-\{0\}}$ such that

$$
\|v\|_{\sigma, s}^{2}:=\sum_{k}\left|v_{k}\right|^{2}|k|^{2 s} \mathrm{e}^{2 \sigma|k|}<\infty .
$$

We will identify a 2 periodic function $v$ with its Fourier coefficients defined by (2.24). We will say that $v \in \ell_{\sigma, s}^{2}$ if its Fourier coefficients have this property. Correspondingly we define the phase spaces $\mathcal{P}_{s}$ by

$$
\mathcal{P}_{s}:=\ell_{\sigma, s+1}^{2} \times \ell_{\sigma, s}^{2} \ni(v, u),
$$

and denote

$$
\|(v, u)\|_{s}^{2}:=\|v\|_{\sigma, s+1}^{2}+\|u\|_{\sigma, s}^{2} ;
$$

$\sigma$ is fixed. The ball of radius $R$ centered at the origin of $\mathcal{P}_{s}$ will be denoted by $B_{s}(R)$.

Then the flow $\Psi^{\tau}$ of the system $H_{0}$ is unitary in all the spaces $\mathcal{P}_{s}$.

Theorem 4.2. For any $r \geq 5$ there exists a constant $\mu_{*} \equiv \mu_{* r}$, such that, if

$$
\mu<\mu_{*}
$$

then there exists an analytic canonical transformation $\mathcal{T}: B_{r}(1) \rightarrow B_{r}(2)$ which averages $K$, namely such that

$$
K \circ \mathcal{T}=H_{0}+\mu^{2}\langle P\rangle+\mathcal{R} ;
$$

the vector field $X_{\mathcal{R}}$ of the remainder is analytic in a complex ball of radius 1 and fulfills the estimate

$$
\sup _{\|z\|_{r} \leq 1}\left\|X_{\mathcal{R}}(z)\right\|_{0} \leq C_{r} \mu^{4-\frac{12}{6+r}} .
$$


Moreover for any $1 \leq r_{1} \leq r$ the transformation $\mathcal{T}$ maps $B_{r_{1}}(1)$ into $\mathcal{P}_{r_{1}}$ and fulfills

$$
\sup _{\|z\|_{r_{1}} \leq 1}\|z-\mathcal{T}(z)\|_{r_{1}} \leq C \mu^{2-\frac{6}{6+r}} .
$$

The idea of the proof is to approximate the system by a system with finitely many degrees of freedom, say $M$, to construct the canonical transformation that averages the finite dimensional system, and finally to choose $M$ in a such a way that the order of magnitude of the error due to the averaging procedure is equal to the order of magnitude of the error due to the cutoff procedure. For the details see [BP05].

Theorem 4.2 shows that the equations of motions of the FPU are a perturbations of the normal form equations described in the previous section. However they are a very singular perturbation since the remainder has a vector field which small only when considered as an operator extracting $r$ derivatives, with $r \geq 5$. As pointed out in [Bam05] the use of such a normal form for dynamical previsions is far from trivial. In the present case one can use a technique developed by Schneider and Wayne in [SW00] and generalized to a form suitable for our purpose [BP05, Bam05].

Since when dealing with the FPU system we are interested in Fourier modes, we explicit state their definition. Thus, for a given FPU state $\left(q_{j}, p_{j}\right)$, we have

$$
p_{j}=\frac{1}{\sqrt{2 N}} \sum_{k=-N}^{N-1} \hat{p}_{k} \mathrm{e}^{\mathrm{i} \frac{j k \pi}{N}}
$$

and similarly for $q_{j}$. We denote by

$$
E_{k}:=\frac{\left|\hat{p}_{k}\right|^{2}+\omega_{k}^{2}\left|\hat{q}_{k}\right|^{2}}{2}, \quad k=-N \ldots ., N-1
$$

the energy of the $k$-th mode.

Let $\xi^{a}(y, \tau), \eta^{a}(y, \tau)$ be a solution of the normal form equations (2.34). Correspondingly, we define an approximate solutions $z^{a} \equiv\left(r^{a}, s^{a}\right)$ of the FPU by

$$
\begin{aligned}
& r^{a}(x, t):=\frac{\xi^{a}(\mu x, \mu t)+\eta^{a}(\mu x, \mu t)}{\sqrt{2}} \\
& s_{x}^{a}(x, t):=\frac{\xi^{a}(\mu x, \mu t)-\eta^{a}(\mu x, \mu t)}{\sqrt{2}}
\end{aligned}
$$

and an initial datum for the FPU by $r_{0, j}:=r^{a}(j, 0), s_{0, j}:=s^{a}(j, 0)$. We will denote by $\left(r_{j}(t), s_{j}(t)\right)$ the corresponding true solution of the FPU.

Then we have the following

Theorem 4.3. Fix a large $T_{f}$; assume that for all times $|t|<T_{f} / \mu^{3}$ the approximate solution is such that $\left(\xi^{a}, \eta^{a}\right) \in \mathcal{P}_{78}$ and has norm smaller than 1 and fix some $\sigma>0$. Then there exists $\mu_{*}$ depending on $T_{f}$ and on $\left\|\left(\xi^{a}(t), \eta^{a}(t)\right)\right\|_{78}$ only, such that, if $\mu<\mu_{*}$ then for all times $t$ fulfilling

$$
|t| \leq \frac{T_{f}}{\mu^{3}}
$$


one has

$$
\sup _{j}\left(\left|r_{j}(t)-r^{a}(j, t)\right|+\left|s_{j}(t)-s^{a}(j, t)\right|\right) \leq C \mu \quad ;
$$

moreover

$$
\left|\frac{E_{k}(t)}{N}-\mu^{4}\left[\frac{\left|\hat{\xi}_{K}^{a}(t)\right|^{2}+\left|\hat{\eta}_{K}^{a}(t)\right|^{2}}{2}+\frac{\left|\hat{\xi}_{-K}^{a}(t)\right|^{2}+\left|\hat{\eta}_{-K}^{a}(t)\right|^{2}}{2}\right]\right| \leq C \mu^{5}
$$

for all $k$ such that $\frac{k}{N}=\mu K$ with $|K| \leq \frac{|\ln \mu|}{2 \sigma}$, and

$$
\frac{\left|E_{k}(t)\right|}{N} \leq \mu^{5}
$$

for all $k$ such that $\frac{k}{N}=\mu K$ with $|K|>\frac{|\ln \mu|}{2 \sigma}$, whereas $E_{k}(t)=0$ otherwise.

We recall that the Fourier coefficients $\hat{\xi}_{K}^{a}$, and $\hat{\eta}_{K}^{a}$ are defined by (2.20).

We remark that in view of the discussion of the previous section we can ensure rigorously that correspondingly to smooth initial data the solution of the normal form equation is smooth enough to apply the above theorem in the cases $n=3,4$, while we expect that this is true also in the case $n=5$, and also when $n=6$ provided the energy is small enough. On the contrary we expect that this assumption is violated in the case $n \geq 7$.

In particular in the case $n=3$, using the theory of the KdV equation developed in [KP03], one can prove a precise result for the formation of the packet:

It is convenient to state the result in terms of "specific quantities", thus we will label the modes with the index

$$
\kappa:=\frac{k}{N} ;
$$

correspondingly we denote by

$$
\mathcal{E}_{\kappa}:=\frac{E_{k}}{N}
$$

the specific energy in the mode with index $\kappa$.

Theorem 4.4. Fix a constant $C_{0}$ and a positive (large) time $T_{f}$; then there exist positive constants $\mu_{*}, C_{1}, C_{2}$, dependent only on $C_{0}$ and on $T_{f}$, such that the following holds. Consider an initial datum with

$$
\mathcal{E}_{\kappa_{0}}(0)=C_{0} \mu^{4} \quad,\left.\quad \mathcal{E}_{\kappa}(0) \equiv \mathcal{E}_{\kappa}(t)\right|_{t=0}=0, \quad \forall \kappa \neq \kappa_{0},
$$

and assume $\mu<\mu_{*}$. Then, there exists $\sigma>0$ such that, along the corresponding solution, one has

(i)

$$
\mathcal{E}_{\kappa}(t) \leq \mu^{4} C_{1} \mathrm{e}^{-\sigma \kappa / \mu}+C_{2} \mu^{5}, \quad \text { for }|t| \leq \frac{T_{f}}{\mu^{3}}
$$

for all $\kappa>0$. 
(ii) There exists a sequence of almost periodic functions $\left\{F_{n}\right\}_{n \in \mathbb{N}}$ such that, defining the specific energy distribution

$$
\mathcal{F}_{n \kappa_{0}}=\mu^{4} F_{n}, \quad \mathcal{F}_{\kappa}=0 \text { if } \kappa \neq n \kappa_{0}
$$

one has

$$
\left|\mathcal{E}_{\kappa}(t)-\mathcal{F}_{\kappa}(t)\right| \leq C_{2} \mu^{5}, \quad|t| \leq \frac{T_{f}}{\mu^{3}} .
$$

Remark 4.5. Since $F_{n}(t)$ are almost periodic functions of time their time average defined by

$$
\bar{F}_{n}:=\lim _{T \rightarrow \infty} \frac{1}{T} \int_{0}^{T} F_{n}(t) d t
$$

exists (see e.g. [Fin74]). It follows that up to the error the time average of $\mathcal{E}_{\kappa}(t)$ relaxes to the limit distribution obtained by rescaling $\bar{F}_{n}$ as in (4.18).

Remark 4.6. One can give heuristic arguments to show that the (rescaled) limit distribution $\bar{F}_{n}$ is the same for all initial data in a set of full measure.

\section{References}

[Bam05] D. Bambusi. Galerkin averaging method and Poincaré normal form for some quasilinear PDEs. Preprint. To appear in Ann. Scuola Norm. Sup. Pisa, Cl. Sci., 2005.

[BGG04] L. Berchialla, L. Galgani, and A. Giorgilli. Localization of energy in FPU chains. Discrete Contin. Dyn. Syst., 11(4):855-866, 2004.

[BGP04] L. Berchialla, A. Giorgilli, and S. Paleari. Exponentially long times to equipartition in the thermodynamic limit. Phys. Lett. A, 321:167-172, 2004 .

[BP05] D. Bambusi and A. Ponno. On metastability in FPU. Preprint, to appear in Commun. Math. Phys., 2005.

[Fin74] A. M. Fink. Almost periodic differential equations. Springer-Verlag, Berlin, 1974. Lecture Notes in Mathematics, Vol. 377.

[FPU65] E. Fermi, J.R. Pasta, and S.M. Ulam. Studies of nonlinear problems. In Collected works of E. Fermi, vol.2. Chicago University Press, Chicago, 1965.

[GPP05] A. Giorgilli, S. Paleari, and T. Penati. Local chaotic behaviour in the Fermi-Pasta-Ulam system. Discrete Contin. Dyn. Syst. Ser. B, 5(4):991-1004 (electronic), 2005.

[GS72] L. Galgani and A. Scotti. Planck-like distribution in classical nonlinear mechanics. Phys. Rev. Lett., 28:1173-1176, 1972. 
[KBL03] P. R. Kramer, J. A. Biello, and Y. Lvov. Application of weak turbulence theory to FPU model. Discrete Contin. Dyn. Syst., (suppl.):482491, 2003. Dynamical systems and differential equations (Wilmington, NC, 2002).

[KP03] T. Kappeler and J. Pöschel. KdV \& KAM, volume 45 of Ergebnisse der Mathematik und ihrer Grenzgebiete. 3. Folge. A Series of Modern Surveys in Mathematics [Results in Mathematics and Related Areas. 3rd Series. A Series of Modern Surveys in Mathematics]. SpringerVerlag, Berlin, 2003.

[Mer01] Frank Merle. Existence of blow-up solutions in the energy space for the critical generalized KdV equation. J. Amer. Math. Soc., 14(3):555578 (electronic), 2001.

[MM02] Y. Martel and F. Merle. Stability of blow-up profile and lower bounds for blow-up rate for the critical generalized KdV equation. Ann. of Math. (2), 155(1):235-280, 2002.

[PB05] A. Ponno and D. Bambusi. Energy cascade in Fermi-Pasta-Ulam model. In Symmetry and Perturbation Theory 2004, pages 263-270. World Scientific, 2005.

[Pon03] A. Ponno. Soliton theory and the Fermi-Pasta-Ulam problem in the thermodynamic limit. Europhys. Lett., 64(5):606-612, 2003.

[Rin01] B. Rink. Symmetry and resonance in periodic FPU chains. Comm. Math. Phys., 218(3):665-685, 2001.

[Rin03] B. Rink. Symmetric invariant manifolds in the Fermi-Pasta-Ulam lattice. Phys. D, 175(1-2):31-42, 2003.

[SW00] G. Schneider and C. E. Wayne. Counter-propagating waves on fluid surfaces and the continuum limit of the Fermi-Pasta-Ulam model. In International Conference on Differential Equations, Vol. 1, 2 (Berlin, 1999), pages 390-404. World Sci. Publishing, River Edge, NJ, 2000.

[ZK65] N. J. Zabusky and M. D. Kruskal. Interaction of solitons in a collisionless plasma and the recurrence of initial states. Phys. Rev. Lett., 15:240-243, 1965.

\author{
Dario Bambusi, Antonio Ponno \\ Dipartimento di Matematica \\ Via Saldini 50, 20133 Milano, Italy \\ E-mail: bambusi@mat.unimi.it, ponno@mat.unimi.it \\ web-address: http://users.mat.unimi.it/users/bambusi/
}

